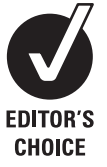

${ }^{1}$ Department of Neuroscience, Section of Clinical Neurophysiology, University of Brescia, Spedali Civili, Italy; ${ }^{2}$ Laboratory of Clinical Epidemiology, Aldo e Cele Daccò Clinical Research Centre, Mario Negri Institute, Ranica (Bergamo), Italy; ${ }^{3}$ Department of Anaesthesia-Intensive Care Medicine, Section of Neuroanestesia and Neurocritical Care, University of Brescia, Spedali Civili, Italy

Correspondence to: Professor N Latronico, Department of AnesthesiologyIntensive Care, Section of

Neuroanestesia and Neurocritical Care, University of Brescia, Piazzale Spedali Civili, 125125 Brescia, Italy; latronic@ med.unibs.it

Received 13 December 2007 Revised 11 February 2008

Accepted 19 February 2008

Published Online First

13 March 2008

\title{
Long-term outcome in patients with critical illness myopathy or neuropathy: the Italian multicentre CRIMYNE study
}

\author{
B Guarneri, ${ }^{1}$ G Bertolini, ${ }^{2}$ N Latronico ${ }^{3}$
}

\begin{abstract}
Background: Critical illness myopathy (CIM) and polyneuropathy (CIP), alone or in combination (CIP/CIM), are frequent complications in patients in the intensive care unit (ICU). There is no evidence that differentiating between CIP and CIM has any impact on patient prognosis.
\end{abstract}

Methods: 1-year prospective cohort study of patients developing CIP, CIM or combined CIP and CIM during ICU stay.

Results: 28 out of 92 (30.4\%) patients developed electrophysiological signs of CIP and/or CIM during their ICU stay, which persisted in 18 patients at ICU discharge. At hospital discharge, diagnoses in the 15 survivors were CIM in six cases, CIP in four, combined CIP and CIM in three and undetermined in two uncooperative patients. During the 1-year follow-up of six patients with CIM, one patient died and five recovered completely within 3 (three patients) to 6 (two patients) months. Of three patients with CIP/CIM, one died, one recovered and one with residual CIP remained tetraplegic. Of four patients with CIP, one recovered, two had persisting muscle weakness and one remained tetraparetic. Conclusion: CIM has a better prognosis than CIP. Differential diagnosis is important to predict long-term outcome in ICU patients.

Acquired neuromuscular disorders are common in patients in the intensive care unit (ICU); incidence in patients with sepsis, multiple organ failure or prolonged ICU stay is $46 \% \quad(95 \%$ confidence interval $43 \%$ to $49 \%){ }^{1}$

Muscle wasting and paralysis are common clinical features, which are ascribed to critical illness polyneuropathy (CIP), critical illness myopathy (CIM) or a combination of CIP and CIM (CIP/CIM). ${ }^{2-4}$

Differential diagnosis between CIP and CIM is often not possible in patients in the ICU because they cannot cooperate for accurate sensory and (in some cases) motor testing, and for conventional needle electromyography (EMG) evaluation, which can help distinguish between the two entities but only with fully cooperative patients. ${ }^{5}$ Recent research has shown that diagnosis of CIM in patients in the ICU can be established by a combination of needle EMG, direct muscle stimulation and plasma creatine kinase. ${ }^{6}$ However, there is no evidence that differentiating between CIP and CIM has any impact on patient prognosis.

We conducted a multicentre prospective study in 92 critically ill adult patients admitted to nine Italian ICUs, and followed them with serial clinical and neurophysiological investigations during their ICU stay and for 1 year after acute care hospital discharge. Results on the acute phase have been reported previously. ${ }^{7}$ We now report the long-term follow-up of diseased patients.

\section{METHODS}

This multicentre prospective cohort study was performed between January 1998 and March 2001 in nine Italian ICUs belonging to the Gruppo Italiano per la Valutazione degli Interventi in Terapia Intensiva (GiViTI). Patients over 15 years of age were included. Written consent was obtained from the patient whenever possible; otherwise, written information was given to their next of kin. Written consent was obtained from all surviving patients as soon as they regained mental competency.

The acronym CRIMYNE (critical illness myopathy and/or neuropathy) identified the current study among the participating centres; this acronym was coined to emphasise that differential diagnosis between CIP and CIM was not a study target during the ICU period.

Twenty-eight patients developed electrophysiological signs of CIP and/or CIM during ICU stay, which persisted in 18 (64.3\%) patients at ICU discharge. At hospital discharge, diagnoses in the 15 survivors were CIM in six cases, CIP in four, combined CIP and CIM in three and undetermined in two uncooperative patients.

\section{Follow-up}

Three, six and 12 months after hospital discharge, patients underwent a four part evaluation to define their degree of clinical recovery: (i) complete neurological examination, (ii) muscle strength evaluation, (iii) global motor performance and (iv) complete electrophysiological investigations of limbs. Investigators who performed the follow-up visits were blind to the diagnosis at hospital discharge.

Muscle strength was evaluated according to the Medical Research Council (MRC) scale. ${ }^{8}$ A test of global motor performance consisted of standing up from a chair, and walking unaided for at least $50 \mathrm{~m}$ with no evidence of ataxia. ${ }^{9}$ Clinical recovery was complete if patients had an MRC grade of $4 / 5$ in all muscles, and successfully performed the global motor performance test. In all other cases, clinical recovery was judged as incomplete, and patients were defined as having muscle weakness; patients with an MRC score of $0-1$ in all four limbs were defined as having tetraplegia or tetraparesis.

Electrophysiological investigations consisted of conventional motor (median and common peroneal nerves) and sensory nerve (median and sural nerves) conduction studies, and needle EMG in the 
Table 1 One-year follow-up data

\begin{tabular}{|c|c|c|c|c|c|}
\hline \multicolumn{6}{|c|}{ Diagnoses on acute care hospital discharge } \\
\hline \multicolumn{6}{|c|}{ CIM 6 patients, CIP 4 patients, combined CIP/CIM 3 patients, undetermined 2 patients (unable to cooperate) } \\
\hline 3 months & 4 recovery & $\begin{array}{l}3 \text { CIM (1 tetraplegia, } \\
1 \text { tetraparesis, } \\
1 \text { muscle weakness) }\end{array}$ & $\begin{array}{l}4 \text { CIP (1 tetraplegia, } \\
1 \text { tetraparesis, } \\
2 \text { muscle weakness) }\end{array}$ & $\begin{array}{l}3 \text { CIP/CIM (1 tetraplegia, } \\
1 \text { tetraparesis, } \\
1 \text { muscle weakness) }\end{array}$ & $\begin{array}{l}1 \text { lost to follow- } \\
\text { up }\end{array}$ \\
\hline \multirow[t]{2}{*}{6 months } & & 2 recovery, 1 death & $\begin{array}{l}4 \text { CIP (1 tetraplegia, } \\
1 \text { tetraparesis }\end{array}$ & 1 recovery & \\
\hline & & & 2 muscle weakness) & $\begin{array}{l}2 \text { CIP/CIM } \\
\text { (1 tetraplegia) }\end{array}$ & \\
\hline \multirow[t]{2}{*}{1 year } & & & 1 recovery & 1 death & \\
\hline & & & $\begin{array}{l}3 \text { CIP ( } 1 \text { tetraparesis, } \\
2 \text { muscle weakness) }\end{array}$ & 1 CIP (1 tetraplegia) & \\
\hline
\end{tabular}

CIM, critical illness myopathy; CIP, critical illness polyneuropathy.

tibialis anterior, quadriceps femori, abductor pollicis brevis and deltoid; details are presented elsewhere.

\section{RESULTS}

Of the 15 patients followed-up, seven were critically ill nonsurgical patients (pneumonia, $\mathrm{n}=2$; pulmonary oedema, pancreatitis, intracerebral haemorrhage, metabolic encephalopathy, post-anoxic encephalopathy, $\mathrm{n}=1$ each), five had multiple trauma and three had head trauma. Twelve patients were male (80\%) and mean age was 44.7 (SD 14.9) years. Mean Simplified Acute Physiology Score II scores in patients with CIM, CIP or CIP/CIM were 41.7 (SD 8.1), 43.5 (7.9) and 46.0 (9.6), respectively. Median Sequential Organ Failure Assessment scores were 9 (range 6-14), 7 (5.0-12.0) and 11.0 (11-12), respectively. Duration of ICU and hospital stay were 24.1 (13.4) days and 53.7 (68.6) days, respectively.

Patients had variable degrees of muscle strength impairment (table 1). Incomplete recovery and persisting disability with tetraparesis and tetraplegia were common. Muscle weakness was symmetrical but recovery of muscle strength was asymmetric in two patients who eventually had unilateral foot drop. Muscle atrophy, reduced or absent deep tendon reflexes and distal sensory loss was observed in the lower limbs of all patients with CIP or CIP/CIM. One patient refused the followup visit and was classified as lost to follow-up; on telephone interview, the patient (a medical doctor) referred he had regained normal muscle strength and had returned to work.

Severity of muscle weakness was not correlated with the clinical and electrophysiological diagnosis but the rapidity and completeness of recovery were. At 3 months, three of the five survivors with CIM had complete clinical and electrophysiological recovery and two patients recovered within 6 months. Conversely, patients with CIP or CIP/CIM had a slower recovery, or did not recover at all.

\section{DISCUSSION}

We found that 15 of 28 (53.6\%) patients developing CIP, CIM or CIP/CIM during their ICU stay had persisting neurological and electrophysiological signs of neuromuscular abnormalities on discharge from the acute care hospital. Of these 15 patients, five $(33.3 \%)$ recovered normal muscle strength and global motor performance within 3 months after hospital discharge whereas 10 (66.7\%) experienced prolonged and severely disabling muscle weakness and paralysis. Patients with a definite diagnosis of CIM recovered earlier and better than those with CIP, the majority of whom remained severely disabled 1 year after hospital discharge.

In a systematic review on long-term outcome, $28.1 \%$ of patients with CIM or CIP had severe disability ${ }^{10}$; mean duration of follow-up was 3-6 months, but the range was extremely variable (2 days to 8 years). Data were insufficient to judge whether different electrophysiological diagnoses were associated with different outcomes. Therefore, this study is the first, to our knowledge, indicating that differential diagnosis of CIP and CIM based on accurate clinical evaluation and complete electrophysiological testing is important to define the risk of prolonged disability after ICU discharge. Clinical evaluation alone is not helpful in this respect as both CIP and CIM may cause profound muscle weakness and paralysis.

Our results are different from those of Lacomis et al who, in a retrospective series, found similar functional outcomes in patients with CIM and CIP. ${ }^{11}$ A possible explanation is that patients where followed-up for only 4 months, when CIM is still largely prevalent. Our data, which need to be confirmed in larger series, suggest that the longer the interval between hospital discharge and electrophysiological examination, the higher the probability that CIM has resolved. This might explain the results of Fletcher et al who found electrophysiological findings consistent with previous $\mathrm{CIP}^{12}$ in a series of 22 critically ill patients studied at a median of 43 months (range 12-57) after ICU discharge.

We conclude that CIM has a better prognosis than CIP. Differential diagnosis is important to predict long-term outcome in patients in the ICU. Therefore, comprehensive clinical and electrophysiological investigations should be performed to precisely define the pathological diagnosis of patients discharged from the ICU.

Competing interests: GB and NL are part of the Steering Committee of the GiViTI (Gruppo Italiano per la Valutazione degli Interventi in Terapia Intensiva), which is the recipient of an unconditional grant from AstraZeneca Italia SpA (Basiglio, Italy), Sanofi-Aventis (Paris, France) and Draeger Italia (Corsico, Italy). The other authors declare that they have no competing interests.

Ethics approval: Ethics approval was obtained.

\section{REFERENCES}

1. Stevens RD, Dowdy DW, Michaels RK, et al. Neuromuscular dysfunction acquired in critical illness: a systematic review. Intensive Care Med 2007;33:1876-91.

2. Bolton CF, Gilbert JJ, Hahn AF, et al. Polyneuropathy in critically ill patients. J Neurol Neurosurg Psychiatry 1984;47:1223-31.

3. Latronico N, Fenzi F, Recupero D, et al. Critical illness myopathy and neuropathy. Lancet 1996;347:1579-82.

4. Bolton CF. Neuromuscular manifestations of critical illness. Muscle Nerve 2005;32:140-63.

5. Latronico N, Peli E, Botteri M. Critical illness myopathy and neuropathy. Curr Opin Crit Care 2005;11:126-32.

6. Lefaucheur JP, Nordine T, Rodriguez P, et al. Origin of ICU acquired paresis determined by direct muscle stimulation. J Neurol Neurosurg Psychiatry 2006;77:500-6.

7. Latronico N, Bertolini G, Guarneri B, et al. Simplified electrophysiological evaluation of peripheral nerves in critically ill patients: the Italian multi-centre CRIMYNE study. Crit Care 2007;11:R11. 
8. Medical Research Council. Aids to the examination of the peripheral nervous system. London: HM Stationery Offiec, 1976.

9. Leijten FS, Harinck-de Weerd JE, Poortvliet DC, et al. The role of polyneuropathy in motor convalescence after prolonged mechanical ventilation. JAMA 1995:274:1221-5.

10. Latronico N, Shehu I, Seghelini E. Neuromuscular sequelae of critical illness. Curr Opin Crit Care 2005;11:381-90.

11. Lacomis D, Petrella JT, Giuliani MJ. Causes of neuromuscular weakness in the intensive care unit: a study of ninety-two patients. Muscle Nerve 1998;21:610-17.

12. Fletcher SN, Kennedy DD, Ghosh IR, et al. Persistent neuromuscular and neurophysiologic abnormalities in long-term survivors of prolonged critical illness. Crit Care Med 2003;31:1012-16

\section{APPENDIX}

\section{The CRIMYNE study}

\section{Participating centres (all in Italy)}

(1) Nicola Latronico, MD, Istituto di Anestesia e Rianimazione; Bruno Guarneri, MD, Servizio di Neurofisiopatologia, Università di Brescia, Spedali Civili, Brescia. (2) Alessandra Tanfani, MD, Luigi Targa, MD, Unità Operativa di Anestesia e
Rianimazione: Chiara Minardi, MD, Fabrizio Rasi, MD, Divisione di Neurologia Ospedale Maurizio Bufalini, Cesena. (3) Diletta Guarducci, MD, Simona Cardona, MD, Unità Operativa di Anestesia e Rianimazione; Lucia Toscani, MD, Tiziana Furlan, Technician, Servizio di Neurofisiopatologia, Ospedale SS Annunziata-USL 10/H, Firenze. (4) Anna Piccioli, MD, Sante Ferrarello, MD, Unità Operativa di Anestesia e Rianimazione I; Aldo Amantini, MD, Antonello Grippo, MD, Servizio di Neurofisiopatologia, Università di Firenze, Azienda Ospedaliera Careggi, Firenze. (5) Renata Pinzani, MD, Dorino Salami, MD, Unità Operativa di Anestesia e Rianimazione: Gian Andrea Ottonello, MD, Gianna Zocchi, Technician, Ospedale San Martino, Genova. (6) Martin Langer, MD, Francesca Ricciardi, MD, II Unità Operativa di Anestesia e Rianimazione; Tullio Mille, MD, Clinica Neurochirurgica, Policlinico S. Matteo, Pavia. (7) Vincenzo Emmi, MD, Giuseppe Rodi, MD, I Unità Operativa di Anestesia e Rianimazione; Tullio Mille, MD, Clinica Neurochirurgica, Policlinico S Matteo, Pavia. (8) Walter Bottari, MD, Roberto Martini, MD, Unità Operativa di Anestesia e Rianimazione; Rossella Sabadini, MD, Luisa Motti, MD, Clinica Neurologica, Arcispedale Santa Maria Nuova, Reggio Emilia. (9) Anselmo Caricato, MD, Francesco Della Corte, MD, Istituto di Anestesia e Rianimazione; Francesca Odoardi, MD, Mauro Lomonaco, MD, Istituto di Neurologia, Università Cattolica Sacro Cuore, Policlinico Gemelli, Roma

\section{Historical note}

\section{Richard Bright and epilepsy}

Before the work of Todd, Hughlings Jackson, and Herpin, Richard Bright in the 1830s was largely responsible for the notion of a cortical basis for epilepsy, which opposed the established view that epilepsy originated in the medulla. Bright described several partial and complex partial seizure patterns. $\mathrm{He}$ also located epileptogenic lesions in the grey (cineritious) matter whence they might be transferred to produce disordered and involuntary motions.

When Richard Bright ${ }^{1}$ began clinical practice, the prevailing idea was that epilepsy was essentially a rapidly reversible disorder of consciousness, the genesis of which lay in the medulla. After the primitive notions of divine, diabolical and mystical causes had waned, epilepsy was considered an idiopathic disease without discernible cause. The concept of cortical epileptogenesis was largely initiated by Bright (1789_ 1858), in 1831, and was later developed by Robert Bentley Todd (1809-1860) in his 1849 account of post-epileptic hemiplegia. ${ }^{2}$ It was rediscovered, quite independently, by Samuel Wilks (1824-1911) in 1866. ${ }^{45}$

When an underlying cause was suspected, attacks were labelled epileptiform (now called symptomatic epilepsy). As a result of minute bedside observations, Bright corrected many erroneous notions of his day and distinguished several clinical patterns of epilepsy, notably some 30 years before Herpin's (1799-1865) ${ }^{6}$ and Hughlings Jackson's (1834-1911) ${ }^{7}$ invaluable papers.

Bright's 1831 text, Diseases of the brain and nervous system (vol 2), ${ }^{8}$ contains 25 elegant coloured plates, and neuropathology detailed for more than 200 patients. It gives a clear account of what we now call absences and temporal or frontotemporal complex partial seizures:

it is simply a momentary absence of mind, the eye is fixed as in thought, yet gazing vacantly, no convulsion, no sound, the occupations of the hand ceases, while the mind for a moment is annihilated; the cloud passes off, the intellect returns, and often, unconscious that its operation has been suspended, the patient resumes the occupation in which he was engaged. At other times, this loss of mind is connected with a slight appearance of convulsive or involuntary action."

His account of cases CCLI and CCLII closely resembles focal epilepsy or simple partial seizures without loss of consciousness.

Infantile convulsions in Bright's time were regarded as symptomatic of some systemic irritability often accompanied by fever, the cause being "most of the [infectious] diseases of infancy", 9 as distinct from epilepsy, which was often of unknown cause. Bright, however, taught that infantile convulsions and epilepsy were the same disorder.

Nearly 20 years later, Robert Bentley Todd published Diseases of the brain and other affections of the nervous system $(1855)^{10}$ and edited the 6000 page Cyclopaedia of anatomy and physiology in 5 volumes (1835-1859), ${ }^{11}$ celebrated for its scientific originality. Todd distinguished "three kinds of convulsions"-the jactitating or choreic, tetanic or tonic, and clonic or epileptiform-but he is best known for his account of post-epileptic paralysis (Todd's paralysis), described in the Lumleian Lecture, ${ }^{1}$ which implicated post-epileptic neuronal exhaustion in "The part of the encephalon primarily disturbed,... the hemispheric lobes".

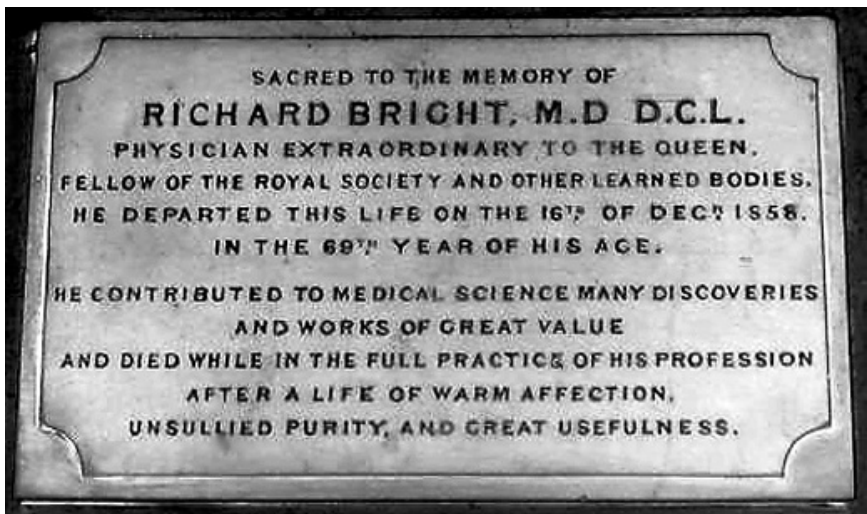

Figure 1 Mural inscription in (Christopher Wren's) St James's Church, Piccadilly. 